\title{
Systemic ALCL Treated in Routine Clinical Practice: Outcomes Following First-Line Chemotherapy from a Multicentre Cohort
}

\author{
Nicolas Martinez-Calle (D) - Amy A. Kirkwood - Maxine Lamb - Alex Smith · Jahanzaib Khwaja $\cdot$ Kate Manos • \\ Caroline Shrubsole • Nicola Gray · Katharine Lewis · Ann Tivey • Mark J. Bishton • Eliza Hawkes • \\ Matthew J. Ahearne $\cdot$ Wendy Osborne $\cdot$ Graham P. Collins · Timothy Illidge $\cdot$ Kim M. Linton · Kate Cwynarski • \\ Cathy Burton · Christopher P. Fox
}

Received: March 7, 2021 / Accepted: April 28, 2021 / Published online: May 26, 2021

(C) The Author(s) 2021

\section{ABSTRACT}

Introduction: Brentuximab vedotin (BV)-CHP is the new standard regimen for first-line treatment of systemic anaplastic large cell lymphoma (sALCL). We undertook a retrospective analysis of consecutive patients diagnosed with

Supplementary Information The online version contains supplementary material available at https:// doi.org/10.1007/s12325-021-01764-0.

N. Martinez-Calle $(\bowtie) \cdot$ M. J. Bishton · C. P. Fox Russell Centre for Clinical Haematology,

Nottingham University Hospitals NHS Trust, City

Hospital Campus, Hucknall Road, Nottingham NG5

1PB, UK

e-mail: n.martinez-calle@nhs.net

A. A. Kirkwood

CRUK and UCL Cancer Trials Centre, UCL Cancer Institute, London, UK

M. Lamb · A. Smith

Haematological Malignancy Research Network,

University of York, York, UK

J. Khwaja · K. Cwynarski

University College of London Hospitals, NHS

Foundation Trust, London, UK

K. Manos

Austin Health, Heidelberg, Australia

C. Shrubsole · W. Osborne

Newcastle Upon Tyne Hospitals NHS Foundation

Trust, Newcastle, UK
sALCL, treated in routine practice, to serve as a benchmark analysis for comparison BV-CHP efficacy in routine practice.

Methods: Patients aged 16 years or older with sALCL treated in seven UK and Australian centres and from 14 additional centres from the UK Haematological Malignancy Research Network database $(n=214)$. Treatment allocation was clinician choice and included best supportive care (BSC). Main outcomes were time to treatment failure (TTF) and overall survival (OS).

\section{N. Gray · G. P. Collins}

Oxford University Hospitals NHS Foundation Trust, Oxford, UK

K. Lewis

Sir Charles Gairdner Hospital, Perth, Australia

\section{A. Tivey $\cdot$ T. Illidge $\cdot$ K. M. Linton}

Cancer Sciences, University of Manchester and the Christie, NHS Foundation Trust, Manchester, UK

\section{E. Hawkes}

Olivia Newton-John Cancer Research Institute,

Austin, Australia

\section{J. Ahearne}

University Hospitals of Leicester NHS Trust, Leicester, UK

C. Burton

Leeds Teaching Hospitals NHS Trust, Leeds, UK 
Multivariable analysis for predictors of both TTF and OS was also undertaken.

Results: The median age 52 years (range 16-93), 18\% ECOG $\geq 3$ and $40 \%$ of cases were ALK positive. CHOP (cyclophosphamide, adriamycin, vincristine, prednisolone) was employed in $152(71 \%)$ of patients and CHOEP (CHOP + etoposide) in $4 \%$ of patients. For CHOP-treated patients overall response rate (ORR) was $65 \%$ and complete response (CR) $47 \%$. Only $9 \%$ of patients underwent autologous stem cell transplant (ASCT). With 57 months median follow-up, 4-year TTF and OS were $41.2 \%$ (95\% CI 33.1-49.1) and 58.9\% (95\% CI 50.3-66.5) respectively. Multivariable analysis showed ALK+ status was independently associated with superior TTF (HR 0.36, 95\% CI 0.21-0.63) but not OS (0.44, 95\% CI 0.18-1.07).

Discussion: We present a retrospective analysis with mature follow-up of one of the largest multicentre populations of sALCL available, comparable to similar large retrospective studies. ALK status remains a strong predictor of outcomes.

Conclusion: These data serve as a robust benchmark for BV-CHP as the new standard of care for sALCL. Similar real-world evidence with BV-CHP will be desirable to confirm the findings of ECHELON-2.

Keywords: Adcetris; Autologous stem cell transplantation; Brentuximab vedotin; CHOEP; CHOP

\section{Key Summary Points}

We report one of the largest observational sALCL studies demonstrating CHOP as the preferred treatment regimen for sALCL. CHOP demonstrated slightly inferior outcomes in the real-world setting when compared to clinical trial data.

Low rates of high-dose therapy (HDT)ASCT performed in CR1 were observed in ALK-negative patients with sALCL in line with current controversy of the true benefit of this strategy.
ALK status remains a significant and strong predictor of outcomes in SALCL, with a $64 \%$ reduction in the risk of death and non-significant 56\% reduction in risk of treatment failure for ALK+ patients.

We also describe the real-world outcomes of $\mathrm{BV}$ in the relapse setting, which compare unfavourably with results from pivotal trials, suggesting a degree of patient selection.

\section{DIGITAL FEATURES}

This article is published with digital features, including a summary slide, to facilitate understanding of the article. To view digital features for this article go to https://doi.org/10.6084/ m9.figshare.14495121.

\section{INTRODUCTION}

Peripheral T cell lymphomas (PTCLs) represent $10-15 \%$ of non-Hodgkin lymphomas and comprise 23 different entities [1]. Anaplastic large cell lymphoma (sALCL) is a relatively rare subtype of T cell non-Hodgkin lymphoma [2], with a prevalence of $12.1 \%$ among PTCLs entities [1]. sALCL is characterized by ALK protein expression in $40-50 \%$ of systemic cases [3]. Cyclophosphamide, adriamycin, vincristine, prednisolone (CHOP) has been the standard of care for frontline sALCL for decades, delivering 5 -year overall survival (OS) rates of $70-81 \%$ for ALK-positive cases [3-5] and 42-49\% for ALKnegative cases [4-6]. The International $\mathrm{T}$ cell Project has reported results of 235 ALK-negative patients with sALCL, describing overall response rate (ORR) and complete response (CR) rates of $77 \%$ and $63 \%$ respectively, median progression free survival (PFS) of 41 months (95\% CI 17-62) and 5-year OS rate of 49\% (95\% CI 35-59) $[7,8]$. The addition of etoposide to CHOP (CHOEP) has been suggested to be superior to CHOP in ALK-positive SALCL. The Nordic Lymphoma Group study [9] found a $66 \%$ 
reduction in risk of death with CHOEP for ALKpositive SALCL, in multivariable cox regression ( $n=97 ; \mathrm{HR}=0.34 ; P=0.008)$. More recently, the German High-Grade non-Hodgkin's lymphoma Study Group (DSHNHL) replicated these findings in ALK-positive sALCL with a 5-year OS of $97 \%$ and a risk reduction of death of $76 \%$ with CHOEP when compared to CHOP (HR $0.24, p=0.002$ ), independent of International Prognostic Index (IPI) and age [10]. For ALKnegative sALCL, a large, single-arm phase II study by the Nordic Lymphoma Group showed an overall response rate of $82 \%$ with CHOEP-14 with $72 \%$ of patients undergoing autologous stem cell transplantation. The 5-year PFS and OS were $44 \%$ and $51 \%$ respectively [11].

More intensive approaches have also been evaluated for sALCL with no significant improvements to the historical CHOP results. A phase II trial reported hyperCVIDD/MA (cyclophosphamide, pegylated doxorubicin, vincristine, dexamethasone alternating with highdose methotrexate/cytarabine) with a CR rate of 83\% and 3-year PFS was 43\% in ALK-negative patients with ALCL [12].

sALCL is characterized by $100 \%$ CD30 antigen cell surface receptor expression. The antiCD30 antibody drug conjugate, brentuximab vedotin (BV) has transformed the treatment of relapsed sALCL following the initial high response rates seen in the phase 1 and 2 trial results leading to durable remission and survival $[13,14]$. More recently, BV was investigated within first-line therapy in the ECHELON-2 trial (NCT01777152) [15], a global randomized phase 3 study of BV in combination with CHP, versus standard CHOP chemotherapy, for newly diagnosed CD30-positive peripheral T cell lymphomas (PTCL). Overall a 34\% reduction in the risk of death for patients with CD30+ PTCL treated with BV-CHP and a $46 \%$ reduction in sALCL populations led to the regulatory approval of A-CHP by both the US Food and Drug Administration (FDA) (for CD30+ PTCL, in 2019) and European Medicines Agency (EMA) (for ALCL only, in 2020).

We undertook a retrospective, multicentre study of newly diagnosed consecutive patients with sALCL, treated prior to the approval of BV$\mathrm{CHP}$, in order to evaluate treatment patterns and outcomes of sALCL in routine clinical practice, where there is a paucity of data. We also evaluated the outcomes of patients treated with single-agent BV for relapsed or refractory $(\mathrm{r} / \mathrm{r})$ ALCL in this large real-world cohort.

\section{METHODS}

Consecutively diagnosed patients with sALCL treated between October 2003 and December 2018 were retrospectively identified from institutional databases at seven haemato-oncology centres in the UK and Australia and 14 additional centres from the UK Haematological Malignancy Research Network database [16] (total patients, $n=214$ ). Inclusion criteria were age at least 16 years old at diagnosis and histological confirmation of sALCL; no central pathology review was undertaken for this study but all cases had undergone expert haematopathology review and fulfilled World Health Organization (WHO) criteria for sALCL. Exclusion criteria included post-mortem diagnosis and participation in the ECHELON-2 trial. Data on DUSP22 and TP63 by fluorescence in situ hybridisation (FISH) were unavailable retrospectively.

Patient baseline characteristics, first and subsequent lines of therapy, BV use and responses in both first-line and subsequent therapy lines were recorded. Response assessment was by CT and/or PET-CT (termed PET hereafter) and assessed by local investigators using standard Deauville score and Lugano classification. Deauville scores $4-5$ were considered as positive. The main outcomes of interest were time to treatment failure (TTF) and OS following first-line and BV treatment. TTF was defined as the time from the start of firstline therapy until the start of subsequent line of therapy, death or progression. Additional outcome measures included frequency of BV use for $\mathrm{r} / \mathrm{r}$ ALCL and frequency of autologous stem cell transplant (ASCT) in first remission. No data on secondary malignancies was collected.

TTF and OS analyses were performed using the Kaplan-Meier method with Cox regression and the log rank test to compare groups in both univariable and multivariable analyses. The 
assumption of proportional hazards was checked using the Schoenfeld residuals, and backwards selection with a $p$ value for inclusion of 0.1 was used to select the final multivariable model. Chi-squared (or Fisher's exact) tests were used to compare frequencies between groups. All statistical analyses were performed using STATA version 15.1 (STATAcorp, Texas).

\section{Compliance with Ethics Guidelines}

All patient data were anonymised at source and treated according to the General Data Protection Regulation (GDPR) and the principles of the declaration of Helsinki. The study was a service evaluation project and exempt from ethic committee approval.

\section{RESULTS}

A total of 231 cases were initially identified, of which 17 were subsequently excluded (14 due to participation in clinical trials and three for post-mortem diagnosis). The final cohort included 214 patients, the median age at diagnosis was 52 years (range 16-93), 135 were male (63\%) and 84 (40\%) patients were ALK-positive. Median age for ALK-positive and ALK-negative was 35 and 61 years respectively. 35 patients (18\%) had Eastern Cooperative Oncology Group (ECOG) performace status $\geq 3$. Baseline patient characteristics are summarized in Table 1 alongside the characteristics of participants of the ECHELON-2 CHOP cohort for comparative purposes.

The majority of patients received multiagent chemotherapy as first-line therapy $(N=191$, $83 \%), 15$ patients (7\%) received palliative treatment only and 7 patients received radiotherapy (RT) only for localized stage disease. Only one patient had missing data for treatment. For those receiving chemotherapy, the median number of cycles administered was 6 (range 1-8) and median lines of therapy received was 2 (range 1-6). The most frequently employed first-line treatment regimen was CHOP in 152 patients (71\%). CHOEP was given to 7 patients (3\%) whereas $27(13 \%)$ received intensified regimens (e.g. CODOX-M/IVAC,
ALCL99). The remaining five patients received other induction regimens (CVP, GCVP [two patients], IVE/methotrexate/DHAP, IVE/ methotrexate). Mortality rate attributed to chemotherapy by investigators was $4 \%(8 / 191)$; all of these patients had received CHOP.

Amongst 198 response-evaluable patients (including those treated with RT alone $n=7$ ), ORR was $71 \%$ and CR rate was $53 \%$. When analysed by ALK status, ALK+ patients had markedly higher CR rates compared to ALKpatients (ALK+ 58\% vs ALK- 37\%, $p=0.01)$ with comparable ORR (ALK+ $67 \%$ vs ALK$63 \%, p=0.59$ ). CHOP-treated patients had an ORR of $65 \%$ and a CR rate of $47 \%$. The seven patients treated with CHOEP and more intensive protocols had an ORR and CR rate of 91\% and $71 \%$ respectively. Twenty-three patients did not have response evaluation, mainly because of death from progressive disease $(n=18$, counted as non-responders) and the remainder because of missing information (excluded).

A minority of patients received ASCT consolidation $(N=20 ; 9 \%), 10$ of whom had received intensified induction regimens and nine had received CHOP. The median IPI of these patients at baseline was 2 (Table 2). The relatively low rate of ASCT was also seen in younger patients in CR1; of 30 ALK-negative patients aged less than 65 years and in CR after initial chemotherapy, only 13 (43\%) underwent ASCT. Of the remaining seven patients who underwent ASCT, two were older than 65 years and five were in partial response (PR). Of note, the relapse rate in patients undergoing ASCT consolidation was $24.5 \%$ (95\% CI 8.6-58.4), as compared to $22.2 \%$ (95\% CI 13.7-34.7) in those potentially eligible (at least 65 years of age and in CR after induction) but who did not undergo ASCT. The International $\mathrm{T}$ cell Lymphoma Project recently reported similar low rates of ASCT (7\%) in ALK-negative ALCL [8].

Median follow-up of was 57 months (IQR 36.3-87.2). The 4-year TTF and OS after first-line treatment were $41.5 \% \quad(95 \%$ CI $34.6-48.2$ months) and 58\% (95\% CI 50.7-64.6 months) respectively (Fig. 1). For the CHOP-treated cohort $(n=152)$, the 4 -year TTF and OS were 41.2\% (95\% CI 33.1-49.1) and 58.9\% (95\% CI 50.3-66.5) (Fig. 1). ALK-negative patients' 
Table 1 Demographic characteristics at baseline of ALCL cohorts and analysed subgroups

\begin{tabular}{|c|c|c|c|}
\hline & All patients $N=214$ & CHOP cohort $N=152$ & ECHELON-2 CHOP cohort $^{\mathrm{c}} N=226$ \\
\hline Age, median (range) & $52.0(16.0-93.0)$ & $52.0(17.0-85.0)$ & $58(18.0-83.0)$ \\
\hline Male, $N(\%)$ & $135(63.1)$ & $94(61.8)$ & $151(67)$ \\
\hline \multicolumn{4}{|l|}{ ECOG, $N(\%)$} \\
\hline $0-1$ & $129(66.5)$ & $94(68.1)$ & $179(79)$ \\
\hline 2 & $30(15.5)$ & $23(16.7)$ & $47(21)$ \\
\hline$\geq 3$ & $35(18.1)$ & $21(15.2)$ & 0 \\
\hline Missing & 20 & 14 & 0 \\
\hline ALK positive, $N(\%)$ & $84(40.0)$ & $67(45.0)$ & $49(22)$ \\
\hline Missing & 4 & 3 & 0 \\
\hline B symptoms, $N(\%)$ & $109(54.2)$ & $77(52.4)$ & $\mathrm{N} / \mathrm{A}$ \\
\hline Missing & 13 & 5 & \\
\hline BM involvement, $N(\%)$ & $14(8.9)$ & $11(9.0)$ & $\mathrm{N} / \mathrm{A}$ \\
\hline Missing & 57 & 30 & \\
\hline \multicolumn{4}{|l|}{ Stage $^{\mathrm{a}}, N(\%)$} \\
\hline I & $22(11.1)$ & $17(11.6)$ & $9(4)$ \\
\hline II & $49(24.7)$ & $38(26.0)$ & $37(16)$ \\
\hline III & $32(16.2)$ & $24(16.4)$ & $67(30)$ \\
\hline IV & $95(48.0)$ & $67(45.9)$ & $113(50)$ \\
\hline Missing & 16 & 6 & \\
\hline$\geq 2$ extranodal sites, $N(\%)$ & $57(27.8)$ & $42(28.4)$ & $\mathrm{N} / \mathrm{A}$ \\
\hline Missing & 9 & 4 & \\
\hline High LDH, $N(\%)$ & $95(54.0)$ & $64(49.6)$ & N/A \\
\hline Missing & 38 & 23 & \\
\hline \multicolumn{4}{|l|}{ IPI score, grouped, $N(\%)^{\mathrm{b}}$} \\
\hline Low: $0-2$ & $119(60.7)$ & $92(63.9)$ & $126(56)$ \\
\hline High: $3+$ & $77(39.3)$ & $52(36.1)$ & $100(44)$ \\
\hline Missing & 18 & 8 & 6 \\
\hline \multicolumn{4}{|l|}{ PIT score, grouped, $N(\%)^{\mathrm{b}}$} \\
\hline Low: $0-1$ & $95(54.6)$ & $74(57.8)$ & $\mathrm{N} / \mathrm{A}$ \\
\hline High: $2+$ & $79(45.4)$ & $54(42.2)$ & N/A \\
\hline
\end{tabular}


Table 1 continued

All patients $N=214 \quad$ CHOP cohort $N=152 \quad$ ECHELON-2 CHOP cohort $^{\mathrm{c}} N=226$

Missing $\quad 40 \quad 24$

$A L C L$ anaplastic large cell lymphoma, $B V$ brentuximab vedotin, $B M$ bone marrow, IPI International Prognostic Index, $L D H$ lactate dehydrogenase, PIT Prognostic Index for T cell Lymphoma

a Stage is based on CT imaging and when available on PET/CT imaging

b Missing scores grouped if definitely low or high regardless of missing values

${ }^{c}$ For comparison

outcomes were inferior compared to ALK-positive with 4-year TTF $28.2 \%$ (95\% CI 18.6-38.6) and OS of $43.9 \%$ (95\% CI 32.6-54.6) vs $57.6 \%$ $(95 \%$ CI $44.8-68.5)$ and $78.6 \% \quad(95 \% \quad$ CI 66.5-86.8), respectively (log rank $p<0.01$ ) (Fig. 2). Multivariable analysis (MVA) for CHOPtreated cohort including a range of established clinical predictive factors (Table 3 ) showed that stage III/IV disease (HR 2.39, 95\% CI 1.22-4.70, $p=0.012$ ), raised LDH (HR $2.18 \quad 95 \%$ CI $1.15-4.11, p=0.016)$ and age (10-year increase) (HR 1.29, 95\% CI 1.04-1.59, $p=0.020$ ) were the only factors independently associated with OS. ALK+ status was not found significant for OS (HR $0.44,95 \%$ CI $0.18-1.07, p=0.071$ ) but retained a significant magnitude effect. ALK+ status was a s ignificantly strong predictor of TTF (HR 0.36, 95\% CI 0.21-0.63, $p<0.001$ ), independently of stage, age and LDH. Additionally, B symptoms (HR 2.45, 95\% CI $1.45-4.15, p=0.001)$ and sex (HR 1.94, 95\% CI $1.16-3.24, p=011$ ) were independently associated with TTF. Baseline demographic characteristics of the complete cases included in the MVA are shown Table 1 in the supplementary material; there were no significant differences between the groups.

Fifty-two patients received $\mathrm{BV}$ at relapse, mostly as second-line therapy $(N=33 ; 63 \%)$. The median number of BV cycles administered was 4 (IQR 1.5-6). The ORR to BV was $49 \%$ and the CR rate was $28 \%$. With a median follow-up after BV was 34 months (IRQ 21.5-50.3), 3-year TTF and OS rates were $29.7 \% \quad(95 \%$ CI 17.5-43.0) and $40.2 \%$ (95\% CI 26.2-53.9) respectively (Fig. 1 in the supplementary material). Twelve patients were bridged to an allogeneic SCT and seven were bridged to ASCT, 15 of these patients remained alive at data cutoff (two allo-SCT and one ASCT patient have died). When analysed by era, $80 \%$ of patients diagnosed in 2013 or beyond had received BV for $\mathrm{r} / \mathrm{r}$ ALCL, as compared to only $53 \%$ of those diagnosed before 2013.

Fifty-three patients were staged with both CT and PET-CT, with 70\% concordance between the two imaging modalities. Ten patients were upstaged with PET-CT, four of whom were found to have extranodal sites not identified by CT. Considering the 175 patients evaluable for treatment response, 95 had PET-CT and 61 had CT only. CR rate was $63 \%$ and $51 \%$ for PET and CT-evaluated patients, respectively. The 3-year TTF was $71.1 \%$ and $71.3 \%$ for PET and CT-defined CR respectively.

\section{DISCUSSION}

The ECHELON-2 study results have changed clinical practice for newly diagnosed sALCL. Data from our study, with mature follow-up, provides a benchmark for the performance of CHOP-like chemotherapy for sALCL treated in routine practice before the approval of BV-CHP. The demonstrated beneficial effect of the BVCHP regimen in the trial setting is likely to be corroborated in patients with sALCL treated with the BV-CHP regimen in the real-world setting.

The survival outcomes for our cohort are comparable to data from the prospective International $\mathrm{T}$ cell Lymphoma Project, which described a 3-year PFS of $47 \%$ in ALK-negative 
Table 2 Demographic characteristics at baseline of ASCT potentially eligible patients aged at most 65 who achieved $\mathrm{CR}$ after induction

\begin{tabular}{|c|c|c|}
\hline & $\begin{array}{l}\text { All patients } \\
N=82\end{array}$ & $\begin{array}{l}\text { ASCT patients } \\
N=13\end{array}$ \\
\hline Age, median (range) & $\begin{array}{l}39.5 \\
\quad(16.0-64.0)\end{array}$ & $43(19-63)$ \\
\hline Male, $N(\%)$ & $49(59.8)$ & $8(62)$ \\
\hline \multicolumn{3}{|l|}{ ECOG, $N(\%)$} \\
\hline $0-1$ & $61(81.3)$ & $7(54)$ \\
\hline 2 & $4(5.3)$ & $1(8)$ \\
\hline$\geq 3$ & $10(13.3)$ & $3(23)$ \\
\hline Missing & 7 & 2 \\
\hline ALK positive, $N(\%)$ & $49(60.5)$ & $0(0)$ \\
\hline Missing & 1 & 0 \\
\hline B symptoms, $N(\%)$ & $39(52.0)$ & $7(54)$ \\
\hline Missing & 7 & $3(23)$ \\
\hline $\begin{array}{l}\text { BM involvement, } N \\
\quad(\%)\end{array}$ & $7(11.1)$ & $5(38)$ \\
\hline Missing & 19 & 4 \\
\hline \multicolumn{3}{|l|}{ Stage $^{\mathrm{a}}, N(\%)$} \\
\hline I & $11(13.9)$ & $2(15)$ \\
\hline II & $26(32.9)$ & $3(23)$ \\
\hline III & $11(13.9)$ & $1(8)$ \\
\hline IV & $31(39.2)$ & $6(46)$ \\
\hline Missing & 3 & 1 \\
\hline $\begin{array}{l}\geq 2 \text { extranodal sites, } \\
\quad N(\%)\end{array}$ & $22(27.8)$ & $5(38)$ \\
\hline Missing & 3 & 1 \\
\hline High LDH, $N(\%)$ & $35(47.9)$ & $8(62)$ \\
\hline Missing & 9 & 1 \\
\hline \multicolumn{3}{|c|}{ IPI score, grouped, $N(\%)^{\mathrm{b}}$} \\
\hline Low: $0-2$ & $60(75.9)$ & $7(54)$ \\
\hline High: $3+$ & $15(22.7)$ & $4(31)$ \\
\hline Missing & 16 & 2 \\
\hline \multicolumn{3}{|c|}{ PIT score, grouped, $N(\%)^{\mathrm{b}}$} \\
\hline
\end{tabular}

Table 2 continued

\begin{tabular}{lll}
\hline & $\begin{array}{l}\text { All patients } \\
\boldsymbol{N}=\mathbf{8 2}\end{array}$ & $\begin{array}{l}\text { ASCT patients } \\
\boldsymbol{N}=\mathbf{1 3}\end{array}$ \\
\hline Low: 0-1 & $51(77.3)$ & $5(54)$ \\
High: 2+ & $15(22.7)$ & $2(15)$ \\
Missing & 16 & 6 \\
\hline
\end{tabular}

a Stage based on CT and when available PET/CT

${ }^{b}$ Missing scores grouped if definitely low or high regardless of missing values

patients with ALCL $[7,8]$. Comparing our findings to CHOP-treated patients in the ECHELON2 trial, recognizing the caveat that $32 \%$ of such patients were of non-ALCL CD30+ PTCL subtypes, the outcomes of our cohort appeared inferior (3-year TTF $43.8 \%$ in our cohort vs $49 \%$ PFS in ECHELON-2). These outcomes were observed despite our CHOP-treated cohort being younger (52 years vs 58 years), with a higher proportion of ALK-positive patients (45\% vs $22 \%$ ) and early stage disease (stage I/II $38 \%$ vs $20 \%$ ). Conversely, consistent with a realworld cohort, our study included $18 \%$ of patients with $\mathrm{ECOG} \geq 3$ (not included in ECHELON -2) which may, at least in part, explain the inferior outcomes (Table 1). ALK status remained as a strong predictor of TTF, independent of age, with ALK-positivity associated with a $64 \%$ reduction in the risk of progression. Given the known association between ALK status and age, it was of no surprise that inclusion of age in the MVA model for OS resulted in a reduction of the significance of ALK status; however, we that believe the magnitude of effect is still clinically significant as there was a $66 \%$ of reduction in risk of death for ALK-positive patients. Our results contrast with those from a French real-world cohort [10] in which an age cutoff of 40 years was associated with outcomes independently of ALK status. By treating age as a continuous variable in our model, the risk of co-segregating age and ALK status is better mitigated; hence, our results do support a strong association of ALK status with clinical outcomes. This effect was evident in our 
A

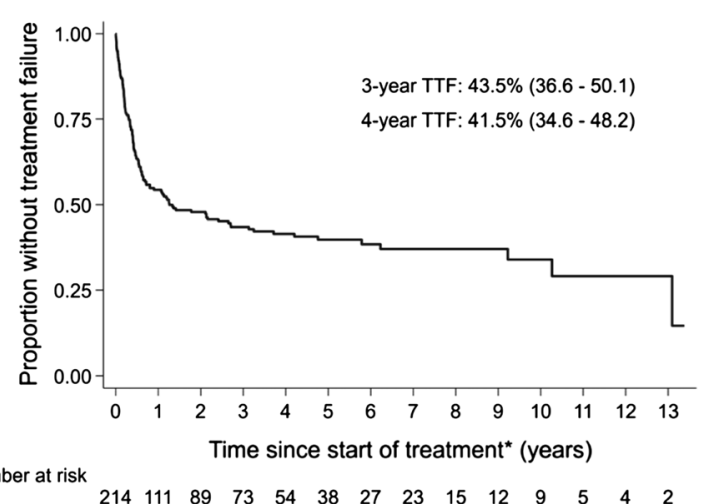

\section{B}
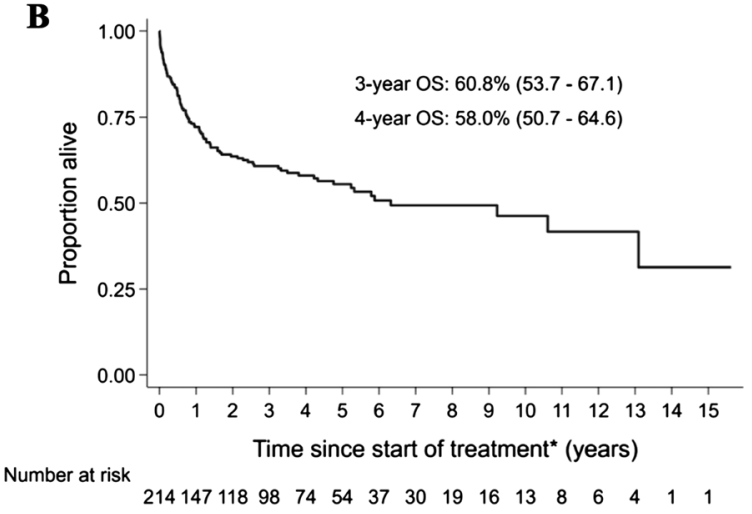

C
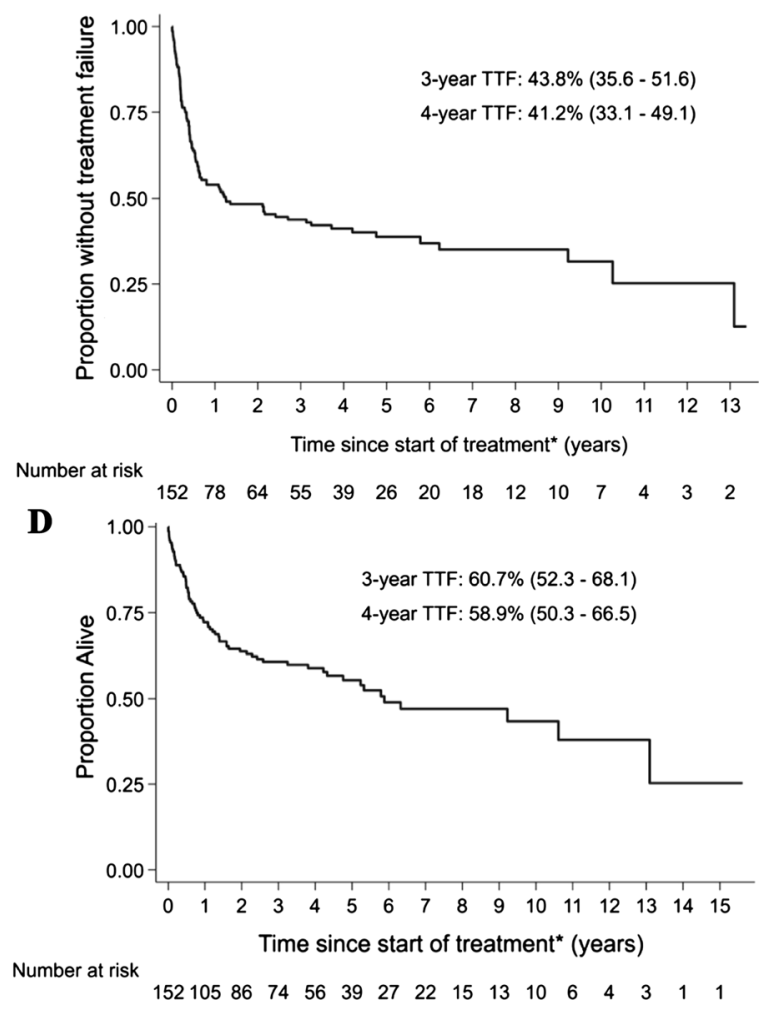

4 Fig. 1 TTF and OS Kaplan-Meier curves of complete cohort $(\mathbf{a}, \mathbf{b})$ and CHOP-treated cohort $(\mathbf{c}, \mathbf{d})$

model, despite differences in median age at diagnosis between ALK+ and ALK- patients.

There are no published randomized trials comparing CHOP with other chemotherapy approaches for newly diagnosed sALCL. Retrospective studies support CHOEP in this context, the Nordic Lymphoma Group study reported improved survival outcomes (HR 0.48, 95\% CI 0.32-0.74) when compared to CHOP in sALCL $[9,17]$. The $\mathrm{T}$ cell lymphoma project also reported a trend towards improved outcomes with etoposide regimens with superior 5-year OS (56\% vs $69 \%$ for etoposide-containing regimens, $p=0.05)$ and a trend towards improved 5 -year PFS (39\% vs 50\% for etoposide-containing regimens, $p=0.18$ ) [8]. However, despite the potential benefit of CHOEP in SALCL, this regimen confers increased risk of morbidity and mortality compared to $\mathrm{CHOP}$, particularly for patients above 60 years of age, as reported in the German NHL-B trial [18]. In our study, there were insufficient numbers of CHOEP-treated patients to analyse this aspect in depth.

Other intensified CHOP-based regimens such as HyperCVAD $[12,19]$ and the CHOP-IVE-MTX regimen (extrapolating from enteropathy-associated $\mathrm{T}$ cell lymphoma protocols [20]) have been applied in ALCL. In our cohort, augmented CHOP regimens were associated with higher CR rates, a higher rate of ASCT consolidation (11/27 patients) and 4-year TTF of $55.7 \%$ (95\% CI 33.8-72.9) and OS of $74.9 \%$ (95\% CI 48.1-89.3). Outcome comparisons with CHOP were not pursued, as the baseline characteristics of these patients were non-comparable and numbers were small.

The role of ASCT for consolidation of firstline therapy in SALCL is controversial [21], although this is included as a recommendation/consideration in some guidelines $[6,22]$. The ECHELON-2 study reported an ASCT rate of $22 \%$ in the $\mathrm{CHP}+\mathrm{BV}$ arm compared to $17 \%$ in the $\mathrm{CHOP}$ arm, reflecting the improved activity of BV-CHP (higher rate of CR) [15]. It is worth 

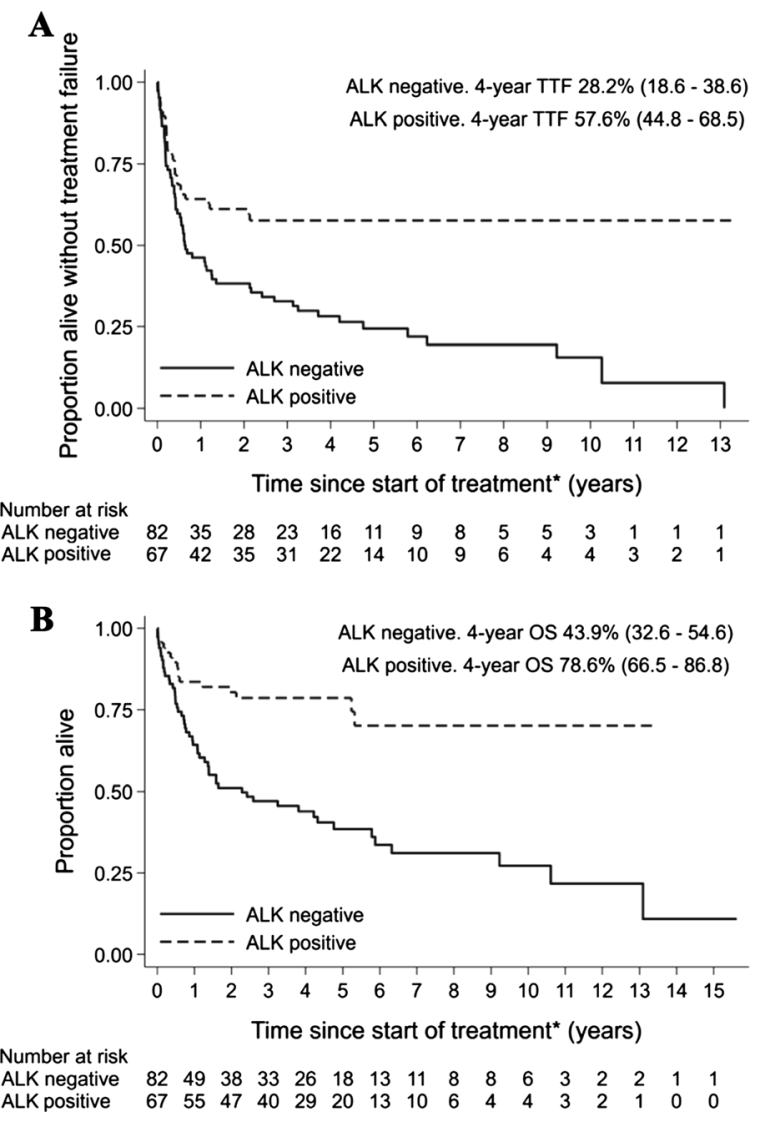

Fig. 2 TTF and OS in CHOP-treated cohort, Kaplan-Meier curves stratified by ALK status

noting that the ASCT intent in the BV-CHP arm of the ECHELON-2 trial from non-Asian countries was $49 \%$ contrasting with $32 \%$ who actually underwent ASCT [23]. There are no randomized studies evaluating ASCT in first response for PTCL or sALCL. A recent multinational retrospective study found no survival advantage of ASCT consolidation as first-line therapy in PTCL [24] with a reported rate of ASCT consolidation of $46 \%$ for sALCL. Conversely, a recent US retrospective real-world study revealed an ASCT rate of only $10 \%$, which is consistent with our cohort, where only $9 \%$ underwent ASCT consolidation [19]. Interestingly, ALK-negative patients with ALCL who underwent ASCT had comparable relapse rates to those in CR who did not. This observation has been replicated by a number of other retrospective studies, with an overall lack of clear benefit of ASCT in first remission $[9,11,19,25-27]$.

As for response evaluation, some studies have established a clear predictive value of response by PET in T cell lymphoma. A retrospective Japanese study $(N=36)$ showed that 3 -year PFS rates in the positive and negative PET patients post induction were $18 \%$ and $62 \%$ respectively [28]. A larger study including 124 patients showed independent OS benefit of PET response with a HR of 3.3 (95\% CI 1.32-8.56) for patients with Deauville $>3$ at end of treatment [29]. Our results suggest that demonstrating CR by either PET or CT is associated with higher rates of TTF at 3 years, underlining the positive impact of chemosensitive disease in the outcomes of sALCL.

\section{CONCLUSIONS}

Our study provides descriptive outcomes of one of the largest multicentre population of sALCL available, confirming ALK-status as a strong and independent predictor of TTF and with nonsignificant but clinically relevant effect on OS. We highlight a low uptake of ASCT consolidation after frontline ALCL therapy, despite being frequently recommended within guidelines and expert recommendations. Our findings likely reflect the uncertainty of evidence supporting this approach. We also highlight the presence of a minority of patients who received BSC from the outset, representing a group with unmet need, for whom novel, low-toxicity and efficacious agents are needed. The present results highlight some degree of patient selection in the pivotal BV trials in the relapsed and frontline setting and serve as an appropriate benchmark for the comparison of patient outcomes treated with frontline BV-CHP. Further research to gather real-world evidence of BV-CHP combination will be desirable. 
Table 3 Univariable and multivariable analyses for OS in the CHOP-treated cohort

\begin{tabular}{|c|c|c|c|c|c|}
\hline & \multicolumn{2}{|c|}{ Complete cases (UVA) $N=92^{\mathrm{a}}$} & \multicolumn{3}{|l|}{ MVA } \\
\hline & Events/ $N$ & HR (95\% CI) & $p$ value & HR (95\% CI) & $p$ value \\
\hline Age (10-year increment) & $47 / 108$ & $1.45(1.21,1.73)$ & 0.0001 & $1.29(1.04-1.59)$ & 0.020 \\
\hline \multicolumn{6}{|l|}{ Sex } \\
\hline Male & $25 / 65$ & 1.00 & 0.24 & - & - \\
\hline Female & $22 / 43$ & $1.41(0.79,2.51)$ & & - & \\
\hline \multicolumn{6}{|l|}{ ECOG } \\
\hline 0 & $13 / 49$ & 1.00 & $0.0010^{*}$ & - & - \\
\hline 1 & $15 / 27$ & $3.34(1.6,7.1)$ & & - & \\
\hline 2 & $19 / 32$ & $3.10(1.5,6.3)$ & & - & \\
\hline \multicolumn{6}{|l|}{ B symptoms } \\
\hline Absent & $23 / 55$ & 1.00 & 0.38 & - & - \\
\hline Present & $24 / 53$ & $1.29(0.73,2.29)$ & & - & \\
\hline \multicolumn{6}{|l|}{ ALK status } \\
\hline Negative & $39 / 61$ & 1.00 & $<0.0001$ & 1.00 & 0.071 \\
\hline Positive & $8 / 47$ & $0.21(0.10,0.45)^{* *}$ & & $0.44(0.18,1.07)$ & \\
\hline \multicolumn{6}{|l|}{ Stage } \\
\hline I-II & $12 / 44$ & 1.00 & 0.0030 & 1.00 & 0.012 \\
\hline III-IV & $35 / 64$ & $2.74(1.41,5.30)$ & & $2.39(1.22,4.70)$ & \\
\hline \multicolumn{6}{|l|}{$\mathrm{LDH}$} \\
\hline Normal & $16 / 58$ & 1.00 & $<0.0001$ & 1.00 & 0.016 \\
\hline Raised & $31 / 50$ & $3.03(1.65,5.58)$ & & $2.18(1.15,4.11)$ & \\
\hline \multicolumn{6}{|l|}{ Extranodal sites } \\
\hline 0 & $16 / 47$ & 1.00 & $0.11^{*}$ & - & - \\
\hline 1 & $17 / 27$ & $2.70(1.4,5.4)$ & & - & \\
\hline $2+$ & $14 / 34$ & $1.67(0.8,3.4)$ & & - & \\
\hline
\end{tabular}

$U V A$ univariable analysis, $M V A$ multivariable analysis

${ }^{*}$ Log-rank test for trend

${ }^{* *}$ Fails the assumption of proportional hazards (MVA model passes)

${ }^{a}$ Demographic characteristics of complete cases are shown in Fig. 1 in the supplementary material 
Table 4 Univariable and multivariable analyses for TTF in the CHOP-treated cohort

\begin{tabular}{|c|c|c|c|c|c|}
\hline & \multicolumn{2}{|c|}{ Complete cases (UVA) $N=108^{\mathrm{a}}$} & \multicolumn{3}{|l|}{ MVA } \\
\hline & Events/N & HR (95\% CI) & $p$ value & HR (95\% CI) & $p$ value \\
\hline Age (10-year increment) & $64 / 108$ & $1.14(0.99,1.32)^{* *}$ & 0.062 & - & - \\
\hline \multicolumn{6}{|l|}{ Sex } \\
\hline Male & $34 / 65$ & 1.00 & 0.074 & 1.00 & 0.011 \\
\hline Female & $30 / 43$ & $1.57(0.96,2.56)$ & & $1.94(1.16-3.24)$ & \\
\hline \multicolumn{6}{|l|}{ ECOG } \\
\hline 0 & $20 / 49$ & 1.00 & $0.0010^{*}$ & - & - \\
\hline 1 & $20 / 27$ & $2.70(1.4,5.1)$ & & - & \\
\hline 2 & $24 / 32$ & $2.56(1.4,4.6)$ & & - & \\
\hline \multicolumn{6}{|l|}{ B symptoms } \\
\hline Absent & $27 / 55$ & 1.00 & 0.016 & 1.00 & 0.001 \\
\hline Present & $37 / 53$ & $1.85(1.12,3.04)$ & & $2.45(1.45-4.15)$ & \\
\hline \multicolumn{6}{|l|}{ ALK status } \\
\hline Negative & $46 / 61$ & 1.00 & 0.0020 & 1.00 & $<0.001$ \\
\hline Positive & $18 / 47$ & $0.42(0.24,0.72)$ & & $0.36(0.21-0.63)$ & \\
\hline \multicolumn{6}{|l|}{ Stage } \\
\hline I-II & $19 / 44$ & 1.00 & 0.0040 & - & - \\
\hline III-IV & $45 / 64$ & $2.20(1.28,3.78)$ & & - & \\
\hline \multicolumn{6}{|l|}{ LDH } \\
\hline Normal & $27 / 58$ & 1.00 & 0.0030 & - & - \\
\hline Raised & $37 / 50$ & $2.14(1.30,3.53)$ & & - & \\
\hline \multicolumn{6}{|l|}{ Extranodal sites } \\
\hline 0 & $23 / 47$ & 1.00 & $0.076^{*}$ & - & - \\
\hline 1 & $20 / 27$ & $2.18(1.2,4.0)$ & & - & \\
\hline $2+$ & $21 / 34$ & $1.63(0.9,3.0)$ & & - & \\
\hline
\end{tabular}

$U V A$ univariable analysis, $M V A$ multivariable analysis

${ }^{*}$ Log-rank test for trend

${ }^{* *}$ Fails the assumption of proportional hazards (MVA model passes)

${ }^{a}$ Demographic characteristics of complete cases are shown in Fig. 1 in the supplementary material 


\section{ACKNOWLEDGEMENTS}

Funding. This study was undertaken as a NHS service evaluation project. The Journal's Rapid Service and Open Access Fee was waived.

Authorship. All named authors meet the International Committee of Medical Journal Editors (ICMJE) criteria for authorship for this article, take responsibility for the integrity of the work as a whole, and have given their approval for this version to be published.

Authors Contributions. Nicolas MartinezCalle, Christopher Fox designed the study, analysed data and wrote the manuscript, Amy Kirkwood performed statistical analysis and reviewed the manuscript. Maxine Lamb, Alexandra Smith, Kate Manos, Caroline Shrubsole, Nicola Gray, Katharine Lewis, Jahanzaib Khwaja, Ann Tivey, Mark Bishton, Eliza Hawkes, Matthew Ahearne, Wendy Osborne, Graham Collins, Kim Linton, Kate Cwynarski, Tim Illidge and Cathy Burton treated patients, contributed to data collection and reviewed the manuscript.

Disclosures. Nicolas Martinez-Calle: Conference travel and accommodation support from Takeda. Kate Manos: Janssen, Honoraria; Novo Nordisk Pharmaceuticals, Travel. Mark Bishton: Takeda, travel support, Research Funding; Janssen, Advisory committees; Celgene, Research Funding; AbbVie, Research Funding; Celltrion, Advisory committees, travel support; Roche, travel support, Research Funding. Eliza Hawkes: Bristol-Myers Squibb, Research Funding, Speakers Bureau; Celgene, advisory committees, research Funding; Mundi pharma, research Funding; Merck Sharp \& Dohme, advisory committees; Roche/Genentech, advisory committees, travel expenses, speakers Bureau; Roche, research Funding; AstraZeneca, Research Funding; Merck KgA, Research Funding; Janssen-Cilag, advisory committees, Research Funding, speakers Bureau; Gilead, advisory committees, research Funding; Takeda, Speakers Bureau. Matthew Ahearne: Takeda, Consultancy and honoraria.
Wendy Osborne: Gilead, Consultancy; Pfizer, honoraria, speakers Bureau; Takeda, consultancy, travel, Speakers Bureau; Novartis, travel; Servier, consultancy; MSD, consultancy; Roche, consultancy, Honoraria, travel, speakers Bureau. Christopher Fox: Remunerated consultant/advisor for Takeda. Graham Collins: COI: Honoraria for advisory and speaker work from Takeda. Kate Cwynarski: Takeda: Consulting/ Advisory Role and Conferences/Travel support. Kim Linton: Beigene, advisory committees; Celgene, advisory committees; Roche, Consultancy; Gilead, advisory committees; Karyopharm, advisory committees; Takeda, Honoraria; Janssen, Travel expenses; Celgene, Travel expenses. Amy Kirkwood, Maxine Lamb, Alexandra Smith, Nicola Gray, Katharine Lewis, Jahanzaib Khwaja, Ann Tivey and Cathy Burton: None to declare. Graham Collins acknowledges support from the Oxford NIHR Biomedical Research Centre and CRUK Experimental Cancer Medicines Centre. Haematological Malignancy Research Network is funded by Cancer Research UK, grant numbers 29685; and Blood Cancer UK, grant number 15037.

Compliance with Ethics Guidelines. All patient data were anonymised at source and treated according to the General Data Protection Regulation (GDPR) and the principles of the declaration of Helsinki. The study was a service evaluation project and exempt from ethic committee approval.

Open Access. This article is licensed under a Creative Commons Attribution-NonCommercial 4.0 International License, which permits any non-commercial use, sharing, adaptation, distribution and reproduction in any medium or format, as long as you give appropriate credit to the original author(s) and the source, provide a link to the Creative Commons licence, and indicate if changes were made. The images or other third party material in this article are included in the article's Creative Commons licence, unless indicated otherwise in a credit line to the material. If material is not included in the article's Creative Commons licence and your intended use is not permitted by statutory regulation or exceeds the 
permitted use, you will need to obtain permission directly from the copyright holder. To view a copy of this licence, visit http:// creativecommons.org/licenses/by-nc/4.0/.

\section{REFERENCES}

1. Moskowitz AJ, Lunning MA, Horwitz SM. How I treat the peripheral T-cell lymphomas. Blood. 2014;17(123):2636-44.

2. Sibon D, Fournier $\mathrm{M}$, Briere J, et al. Long-term outcome of adults with systemic anaplastic largecell lymphoma treated within the Groupe d'Etude des Lymphomes de l'Adulte trials. J Clin Oncol. 2012;32(30):3939-46.

3. Savage KJ, Harris NL, Vose JM, et al. ALK- anaplastic large-cell lymphoma is clinically and immunophenotypically different from both ALK+ ALCL and peripheral T-cell lymphoma, not otherwise specified: report from the International Peripheral T-Cell Lymphoma Project. Blood. 2008;12(111):5496-504.

4. Gleeson M, Peckitt C, Cunningham D, et al. Outcomes following front-line chemotherapy in peripheral T-cell lymphoma: 10-year experience at The Royal Marsden and The Christie Hospital. Leuk Lymphoma. 2018;7(59):1586-95.

5. Savage KJ, Chhanabhai M, Gascoyne RD, et al. Characterization of peripheral T-cell lymphomas in a single North American institution by the WHO classification. Ann Oncol. 2004;10(15):1467-75.

6. Dearden CE, Johnson R, Pettengell R, et al. Guidelines for the management of mature T-cell and NKcell neoplasms (excluding cutaneous T-cell lymphoma). Br J Haematol. 2011;4(153):451-85.

7. Shustov A, Cabrera M, Bellei M, et al. Anaplastic large cell lymphoma, ALK-negative: analysis of 235 cases collected by the T-cell project. Hematol Oncol. 2019;S2(37):283-4.

8. Shustov A, Cabrera ME, Civallero M, et al. ALKnegative anaplastic large cell lymphoma: features and outcomes of 235 patients from the International T-Cell Project. Blood Adv. 2021;3(5):640-8.

9. Cederleuf H, Bjerregard Pedersen M, Jerkeman M, et al. The addition of etoposide to CHOP is associated with improved outcome in ALK+ adult anaplastic large cell lymphoma: a Nordic Lymphoma Group study. Br J Haematol. 2017;5(178): 739-46.
10. Sibon D, Nguyen DP, Schmitz N, et al. ALK-positive anaplastic large-cell lymphoma in adults: an individual patient data pooled analysis of 263 patients. Haematologica. 2019;12(104):e562-5.

11. d'Amore F, Relander T, Lauritzsen GF, et al. Upfront autologous stem-cell transplantation in peripheral T-cell lymphoma: NLG-T-01. J Clin Oncol. 2012;25(30):3093-9.

12. Chihara D, Pro B, Loghavi S, et al. Phase II study of HCVIDD/MA in patients with newly diagnosed peripheral T-cell lymphoma. $\mathrm{Br} \mathrm{J}$ Haematol. 2015;4(171):509-16.

13. Pro $B$, Advani R, Brice $P$, et al. Brentuximab vedotin (SGN-35) in patients with relapsed or refractory systemic anaplastic large-cell lymphoma: results of a phase II study. J Clin Oncol. 2012;18(30):2190-6.

14. Fanale MA, Horwitz SM, Forero-Torres A, et al. Fiveyear outcomes for frontline brentuximab vedotin with CHP for CD30-expressing peripheral T-cell lymphomas. Blood. 2018;19(131):2120-4.

15. Horwitz S, O'Connor OA, Pro B, et al. Brentuximab vedotin with chemotherapy for CD30-positive peripheral T-cell lymphoma (ECHELON-2): a global, double-blind, randomised, phase 3 trial. Lancet. 2019;10168(393):229-40.

16. Smith A, Crouch S, Lax S, et al. Lymphoma incidence, survival and prevalence 2004-2014: sub-type analyses from the UK's Haematological Malignancy Research Network. Br J Cancer. 2015;9(112): 1575-84.

17. Cederleuf $\mathrm{H}$, Pedersen $\mathrm{MB}$, Jerkeman $\mathrm{M}$, et al. Addition of etoposide to CHOP is associated with improved outcome in adult anaplastic large cell lymphoma patients: a Nordic Lymphoma Group Study. Blood. 2015;23(126):340.

18. Wunderlich A, Kloess M, Reiser M, et al. Practicability and acute haematological toxicity of 2- and 3-weekly CHOP and CHOEP chemotherapy for aggressive non-Hodgkin's lymphoma: results from the NHL-B trial of the German High-Grade NonHodgkin's Lymphoma Study Group (DSHNHL). Ann Oncol. 2003;6(14):881-93.

19. Abramson JS, Feldman T, Kroll-Desrosiers AR, et al. Peripheral T-cell lymphomas in a large US multicenter cohort: prognostication in the modern era including impact of frontline therapy. Ann Oncol. 2014;11(25):2211-7.

20. Sieniawski M, Angamuthu N, Boyd K, et al. Evaluation of enteropathy-associated T-cell lymphoma comparing standard therapies with a novel regimen including autologous stem cell transplantation. Blood. 2010;18(115):3664-70. 
21. Corradini P, Marchetti M, Barosi G, et al. SIE-SIESGITMO guidelines for the management of adult peripheral T- and NK-cell lymphomas, excluding mature T-cell leukaemias. Ann Oncol. 2014;12(25): $2339-50$

22. Kharfan-Dabaja MA, Kumar A, Ayala E, et al. Clinical practice recommendations on indication and timing of hematopoietic cell transplantation in mature T Cell and NK/T cell lymphomas: an international collaborative effort on behalf of the Guidelines Committee of the American Society for Blood and Marrow Transplantation. Biol Blood Marrow Transplant. 2017;11(23):1826-38.

23. Savage KJ, Horwitz SM, Advani RH, et al. An exploratory analysis of brentuximab vedotin plus $\mathrm{CHP}(\mathrm{A}+\mathrm{CHP})$ in the frontline treatment of patients with CD30+ peripheral T-cell lymphomas (ECHELON-2): impact of consolidative stem cell transplant. Blood. 2019;Supplement_1(134):464.

24. Fossard G, Broussais F, Coelho I, et al. Role of upfront autologous stem-cell transplantation in peripheral T-cell lymphoma for patients in response after induction: an analysis of patients from LYSA centers. Ann Oncol. 2018;3(29):715-23.

25. Mercadal S, Briones J, Xicoy $\mathrm{B}$, et al. Intensive chemotherapy (high-dose CHOP/ESHAP regimen) followed by autologous stem-cell transplantation in previously untreated patients with peripheral T-cell lymphoma. Ann Oncol. 2008;5(19):958-63.

26. Yam C, Landsburg DJ, Nead KT, et al. Autologous stem cell transplantation in first complete remission may not extend progression-free survival in patients with peripheral $\mathrm{T}$ cell lymphomas. Am J Hematol. 2016;7(91):672-6.

27. Park SI, Horwitz SM, Foss FM, et al. The role of autologous stem cell transplantation in patients with nodal peripheral T-cell lymphomas in first complete remission: report from COMPLETE, a prospective, multicenter cohort study. Cancer. 2019;9(125):1507-17.

28. N, Tomita, Hattori Y, Fujisawa S, et al. Post-therapy ${ }^{18} \mathrm{~F}$-fluorodeoxyglucose positron emission tomography for predicting outcome in patients with peripheral $\mathrm{T}$ cell lymphoma. Ann Hematol. 2015;3(94):431-6.

29. El-Galaly TC, Pedersen MB, Hutchings $M$, et al. Utility of interim and end-of-treatment PET/CT in peripheral T-cell lymphomas: a review of 124 patients. Am J Hematol. 2015;11(90):975-80. 